\title{
MRS MEMBERSHIP APPLICATION MRIS
}

(please print)

Please enter my membership in the Materials Research Society for 1986.

Membership category:

\$45.00 Regular Member (includes subscription to MRS BULLETIN and Journal of Materials Research).

\$25 of the Regular Membership dues is allocated for subscription to Journal of Materials Research.

$\$ 15.00$ Student Member (includes subscription to MRS BULLETIN).

Students must enclose evidence of university registration.

Students may subscribe to Journal of Materials Research for an additional $\$ 15$.

NAME

\begin{tabular}{lll}
\hline Last & First/M.I. \\
\hline
\end{tabular}

POSITION/TITLE

INSTITUTION

ADDRESS

street and/or p.o. box

city

state/province

zip/postal code

country

This address is: $\square$ Institution $\square$ Home

BUSINESS TELEPHONE ( $\underset{\text { area code }}{(-})$ number

RESEARCH INTERESTS

NAMES AND ADDRESSES OF COLLEAGUES WHO MAY BE INTERESTED IN THE MATERIALS RESEARCH SOCIETY:

Please complete this form and return with check* or money order payable to Materials Research Society to:

Materials Research Society

9800 McKnight Road, Suite 327

Pittsburgh, Pennsylvania 15237 USA

"Payment from outside the United States should be in U. S. Dollars and should be drawn on a correspondent U. S. Bank. 


\section{SEMICONDUCTOR-BASED HETEROSTRUCTURES: \\ INTERFACIAL STRUCTURE \& STABILITY}

May 1-2, $1986 \bullet$ AT\&T Bell Laboratories, Murray Hill, New Jersey

Approximately 30 papers ( 9 invited) will be presented at the 1986 Northeast Regional Meeting, addressing Semiconductor-Based Heterostructures and the increasingly significant role they will play in future microelectronic devices.

Presentations in 4 technical sessions will focus on the materials science aspects of interfaces such as perfection, structure and stability, and their relationships to synthesis techniques. Major topics to be highlighted include: Amorphous and Strained-layer superlattices; Si on insulators; Epitaxial Silicides; Compound Semiconductors on Si; Metallization \& Passivation of III-V Compounds; Multilevel metallization schemes for VLSI; Nanocomposite materials; Interfacial \& Pseudomorphic Growth; Ohmic contact to compound semiconductors; Phase stability; and III-V synthesis (MOCVD vs MBE).

Invited presentations will be:

- "Chemical Interactions and Fermi Level Pinning at Metal/III-V Interfaces" L. Brillson, Xerox "Synthesis of Strained-Layer Superlattices by $M O C V D$ ” R. Dupuis, AT\&T Bell Labs "Passivation of III-V Compound Semiconductors" R. Enstrom, RCA

\begin{abstract}
- "Physical \& Chemical
R.F.C. Farrow, IBM
Group IV Elements"

L.

- "Heteroepitaxy of GaAs on Si by MBE"

G.W. Turner, Lincoln Labs

- "MBE Synthesis of Superlattices"

G. Wicks, Cornell
\end{abstract}

Lcture of Semiconductor Interfaces
C. Feldman, AT\&T Bell Labs

- "Current Status of Schottky-Barrier Formation at Silicide-

Silicon Interfaces" P.S. Ho, M. Liehr, IBM

- "Structure \& Thermal Relaxation of Amorphous Semiconductor Interfaces" P. Persans, Exxon
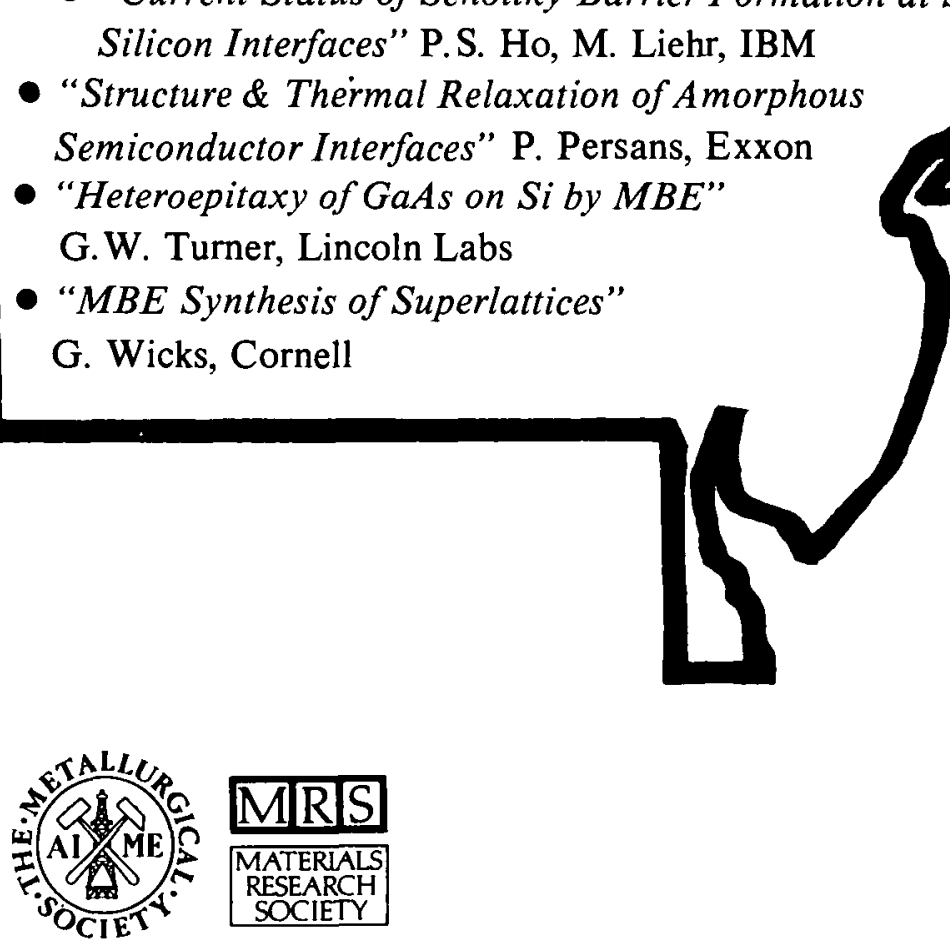

Tutorials and a dinner are being planned to supplement the technical program.

For more information and your advance registration packet, contact:

Meetings Department The Metallurgical Society 420 Commonwealth Drive Warrendale, PA 15086 (412) 776-9050

Telex 9103809397 


\section{MRS CORPORATE AFFILIATES}

AG Associates

Air Products and Chemicals

AIRCO Temeseal

ALCOA

Allied Corporation

American Fly Ash Co.

American Microsystems, Inc.

AMOCO Corporation

Applied Materials

ARCO Metals Company

ARCO Solar, Inc.

AT\&T Bell Laboratories

AT\&T Technologies, Inc.

Blake Industries, Inc.

Branson, IPC

Brimrose Corporation of America

Brush Wellman, Inc.

Cabot Corporation

Cameca Instruments, Inc.

CEMCOM Research Associates

CILAS Alcatel

Combustion Engineering, Inc.-Power Systems

CVC Products, Inc.

Denton Vacuum, Inc.

Drytek, Inc.

E. I. du Pont de Nemours \& Company

DynaVac

Eastman Kodak Company

Eaton Corporation

Electric Power Research Institute

Electronic Space Products International

ELKEM Chemicals, Inc.

Elsevier North-Holland

Energy Conversion Devices, Inc.

Engelhard Corporation

Charles Evans \& Associates

Exxon Research and Engineering Company

Ferranti, plc

GCA Corporation

General Electric Ceramics, Inc.

General Electric Company

General Ionex Corporation

General Motors Research Laboratories

W. R. Grace \& Company

GTE Laboratories

Harshaw/Filtrol

Helionetics

High Voltage Engineering Europa B.V.

Hirst Research Centre

Hitachi Scientific Instruments

Hoya Optics, Inc.

Hughes Aircraft Company

Imperial Chemical Industries, plc

Instruments $\mathrm{SA}$, Inc.

International Business Machines Corporation

International Zeolite Association

JEOL USA

Jet Propulsion Laboratory

Johnson \& Johnson Products, Inc.
Lam Research

Lambda Physik

Lanxide Corporation

Lasertechnics

Lawrence Livermore National Laboratory

Leybold-Heraeus

LFE Corporation

Los Alamos National Laboratory

Lumonics

3M Company

Martin Marietta Laboratories

Materials Research Corporation

Microscience, Inc.

MKS Instruments, Inc.

Mobil-Solar

Monsanto

National Electrostatics Corporation

National Semiconductor

Newport Corporation

Nissei Sangyo America, LTD.

Perkin-Elmer

Philips Electronic Instruments, Inc.

Plasma Technology (UK) Ltd.

Plasma-Therm Systems, Inc.

Portland Cement Association

Pratt \& Whitney

Quantronix Corporation Questek, Inc.

Rockwell International

Rome Air Development

Sandia National Laboratories

Schlumberger-Doll Research

Schott Glass Technologies, Inc. SEH America, Inc.

L. M. Simard, Inc.

SOHIO Chemicals \& Industrial Products Solar Energy Research Institute Solarex

Spex Industries, Inc. Spire Corporation

Surface Science Laboratories, Inc.

Tamarack Scientific Co. Tegal Corporation

Tektronic Inc.

Texas Instruments, Inc.

Tracor Northern, Inc.

TRW

Union Carbide Corporation

United Technologies Research Center

UOP

Varian/Extrion

VG Instruments, Inc.

Wacker Siltronic Corporation

Westinghouse Electric Corporation

Xerox Corporation

XMR

Zimmer

Zymet 